\title{
CONNECTIVITY PROPERTIES FOR SUBSPACES OF FUNCTION SPACES DETERMINED BY FIXED POINTS
}

\author{
DACIBERG L. GONÇALVES AND MICHAEL R. KELLY
}

Received 31 October 2001

We study the topology of a subspace of the function space of continuous selfmappings of a given manifold: the subspace determined by maps having the least number of fixed points in its homotopy class. In the case that the manifold is a closed disk of finite dimension, we prove that this subspace is both globally and locally path connected. We also prove this result when the manifold is a sphere of dimension 1,3 , or 7 .

\section{Introduction}

Let $X$ be a topological space and let $C(X)$ denote the space of continuous selfmaps of $X$ with the compact-open topology. The purpose of this paper is to study the topological properties of the subspace of $C(X)$ where membership is characterized by having the least number of fixed points in a given homotopy class. That is, given $f: X \rightarrow X$, we consider the subspace

$$
C_{\min }(X, f)=\{g \sim f \mid \# \operatorname{Fix}(g)=M F[f]\}
$$

where $M F[\cdot]$ denotes the least number of fixed points possible among all maps in the given homotopy class.

It is of interest to understand the topology of such spaces. In this paper, we only consider the elementary connectedness properties of $C_{\min }(X, f)$ : path connectedness and local path connectedness.

Path connectedness can be reformulated as a 1-parameter problem in fixedpoint theory. That is, given two homotopic maps each having the minimal number of fixed points, we ask about the existence of a homotopy such that each level map has the same property. For local path connectedness, we ask for small homotopies joining two sufficiently close maps. 
Questions and related obstructions for the 1-parameter problem have been studied by a number of authors. Examples of the nonexistence of such homotopies are found in [9]. In [3, 4], obstructions are given to deforming a given homotopy to one with fewer fixed points. In particular, to determine when a given circle of fixed points can be removed. The similar problem for coincidence points of homotopies between two pairs of maps is considered in [7]. A number of papers have appeared dealing with the study of the topology of a variation of $C_{\text {min }}$ which is comprised of the orientation-preserving homeomorphisms of the plane, either having one fixed point (see $[2,14]$ ) or are fixed point free $[1,11,12]$.

In $[5,6]$, the authors study the 1-parameter problem of finding minimal homotopies joining a given pair of minimal surface mappings. One of the results obtained in these works is a connectivity result for the 2-dimensional torus, denoted by T. From [6, Theorems 1.3 and 3.3], we have the following theorem.

Theorem 1.1. The space $C_{\min }(T, f)$ is path connected if and only if the Lefschetz number of $f$ is nonzero.

Moreover, we can check the arguments given in that paper and see that the same characterization holds when we replace path connected with local path connected. The work in this paper is motivated by a result in [5], that for the 2sphere there is a countable family of examples of homotopic maps, each having one fixed point, but any homotopy between the maps must increase the number of fixed points.

In this paper, we consider the two path connectivity problems in a simplyconnected setting. In particular, for the space $X$ being either a disk or a sphere. Moreover, due to the simple nature of the topology of these spaces, we will address dimensions other than two. In Section 2, we study homotopies between maps of the $k$-dimensional disk $D$ which have exactly one fixed point. We establish the result that $C_{\min }(D, c)$ is both path connected and locally path connected (Corollary 2.2). In Section 3, we study the path connected properties in the case of spheres. Using multiplicative structures, we obtain affirmative results in the dimensions 1,3 , and 7 .

\section{Connectivity properties for disks}

The purpose of this section is to give a proof of the two connectedness properties for $C_{\min }(X, f)$ in the case when $X$ is the closed disk of dimension $k$. The result is stated as Corollary 2.2. For the following proposition, and throughout this paper, we use the term Wecken homotopy to mean a homotopy $h_{t}, 0 \leq t \leq 1$, which has the property that $h_{t}$ is in $C_{\min }\left(X, h_{0}\right)$ for each value of $t$. That is, a path in $C_{\min }$ joining $h_{0}$ to $h_{1}$.

Proposition 2.1. Let $f: D \rightarrow D$ be a continuous map of the closed $k$-dimensional disk $D$ which has only one fixed point. Then

(A) there is a Wecken homotopy which connects $f$ to the constant map $c(x)=0$; 
(B) given $\epsilon>0$, there is a $\delta>0$ such that for any $g$ having exactly one fixed point and such that the distance between $f$ and $g$ is less than $\delta$, there is a Wecken homotopy such that the map at each level of the homotopy is distance less than $\epsilon$ from $f$.

Proof. To prove part (A), we consider two cases depending on whether the fixed point of $f$ is in the interior of $D$ or on the boundary of $D$.

We first consider the case where the fixed point $x_{0}$ of $f$ is in the interior. Here we identify $D$ with the unit ball in $R^{k}$ with $\|\cdot\|$ denoting the standard norm. If $x_{0} \neq 0$, let $h_{t}$ be an isotopy of the identity such that $h_{1}$ takes $x_{0}$ to the origin. The composite $h_{t}^{-1} \circ f \circ h_{t}$ gives a Wecken homotopy between $f$ and a map which has the origin as fixed point. So, without loss of generality, we may assume that the fixed point is the origin 0 . Also, by a small Wecken deformation, we can assume that $f$ is inward on the boundary, that is, $\|f(x)\|<\|x\|$ for all $x$ in the boundary of $D$. Consider the family of functions $\phi_{t}(s)=s^{t}$ indexed by $t \in(0,1]$ and each is defined in the closed interval $[0,1]$. Define $\phi_{0}$ to be the limiting function $\phi_{0}(s)=1$ when $s \neq 0$, and $\phi_{0}(0)=0$.

Define a homotopy $H: D \times I \rightarrow D$ by the formula

$$
H(x, t)=\frac{\|x\|}{\phi_{t}(\|x\|)} f\left(\frac{x \phi_{t}(\|x\|)}{\|x\|}\right)
$$

for $x \neq 0$, and $H(0, t)=0$ otherwise. This is well defined because the modulus of $x \phi_{t}(\|x\|) /\|x\|$ is at most $\left\|\phi_{t}(\|x\|)\right\|$. The map $H$ is continuous. This can be seen by noting that the modulus of $H(x, t)$ is bounded above by $\|x\|^{1-t}$ when $t \neq 1$, and by $\|f(x)\|$ in the case $t=1$. Also, for $t$ fixed the level map $H(x, t)$ has only one fixed point for every $t$. This follows from the equality

$$
H(x, t)=x-\frac{\|x\|}{\phi_{t}(\|x\|)}\left(\frac{x \phi_{t}(\|x\|)}{\|x\|}-f\left(\frac{x\|x\|}{\phi_{t}(\|x\|)}\right)\right)
$$

for each $t$. Clearly, $H(\cdot, 1)$ is just the map $f$, while for $t=0$ we have that $H(\cdot, 0)=$ $\|x\| f(x /\|x\|)$. Thus, $H(\cdot, 0)$ is inward mapping for all $x \neq 0$. So we can connect $H(\cdot, 0)$ to the constant map at zero.

The second case, where the fixed point is on the boundary, is proven in a similar manner. In this case, we identify $D$ with the half disk in $R^{k}$. That is the intersection of the unit ball with those points having first coordinate greater than or equal to zero.

The proof of part (B) will be divided into two cases in the same way. Consider first the case where the fixed point of $f$ is in the interior of $D$ which as before is the unit ball. Without loss of generality, we can assume that $\operatorname{fix}(f)=0$.

Choose $\delta_{1}$ so that the distance between $f$ and any $g$ less than $\delta_{1}$ implies that the fixed point of $g$ lies in the ball of radius $\epsilon / 2$. Now given $g$, if it does not have the origin as fixed point, we can deform $g$ by a Wecken homotopy to a function $g^{\prime}$ which has distance from $g$ less than $\epsilon / 2$, and such that $g^{\prime}$ has the origin as the 
fixed point. Hence, consider an isotopy $h_{t}$ of the disk of radius $\epsilon / 2$ relative to the boundary which starts with the identity and ends in a homeomorphism $h_{1}$ which send the fixed point of $g$ to the origin. Take $g^{\prime}$ as the composite $h_{1} \circ g \circ h_{1}^{-1}$.

Now, we assume that both $f$ and $g$ have the origin as the fixed point. Consider the functions $s_{f}, s_{g}:(D-0) \rightarrow\left(R^{k}-0\right)$ defined by $s_{f}(x)=f(x)-x$ and $s_{g}(x)=g(x)-x$. The homotopy class for each of these maps is determined by the degree $S^{k-1} \rightarrow S^{k-1}$ (see, e.g., [13, Theorem 7.4.6]). But $s_{f}$ (and similarly for $\left.s_{g}\right)$ is homotopic to the map $x \mapsto(f(x)-x) /(|f(x)-x|)$. The degree of this map is +1 which is the index of the single fixed point. Hence, $s_{f}$ and $s_{g}$ are homotopic. Moreover, the homotopy extends to $0 \times I$ by mapping to 0 . Now, by adding $x$ to this homotopy, we get a homotopy between $f$ and $g$. We note that in this construction there exists a $\delta_{2}$ such that if the distance from $g$ to $f$ is less than $\delta_{2}$, then each level of the homotopy is within $\epsilon$ of $f$.

The remaining problem is that the image of the homotopy may not lie in $D$. A natural way to resolve this is by radially retracting points outside of $D$ to the boundary of $D$. But in doing so, it is possible that we have introduced fixed points on the boundary of $D$. We choose a $\delta_{3}$ dependent on how $f$ acts on the boundary of $D$. In particular, $\delta_{3}$ is chosen so that for any map $h$ within $\delta_{3}$ of $f$, the image $h(x)$ is never radially outwards from $x$ for each $x$ in the boundary of $D$. Thus, if our homotopy stays within $\delta_{3}$ of $f$, then our above construction has not created any fixed points.

Finally, choosing $\delta$ as the minimum among $\delta_{1}, \delta_{2}$, and $\delta_{3}$ completes the proof in this case. We leave the case where the fixed point is in the boundary to the reader.

As a consequence, we obtain the following corollary. We take, as our definition of locally path connected, that at each point there exists arbitrarily small path connected open sets about the point. So, for completeness, we give Proposition 2.3 and its proof to obtain local connectivity from Proposition 2.1.

Corollary 2.2. The space $C_{\min }(D, c)$ is both path connected and locally path connected.

Proposition 2.3. Let $X$ be a topological space with the property that for each $x \in X$ and each open set $U$ containing $x$, there is an open set $V$ containing $x$ such that each pair of points in $V$ can be joined by a path in $U$. Then, $X$ is locally path connected.

Proof. Fix $x \in U$ as in the proposition. Let $W$ denote the set $\{y \in U \mid$ there is a path in $U$ from $x$ to $y$ \}. First, we show that $W$ is an open set. Let $w \in W$. By hypothesis there is an open set $V$ with $w \in V \subset U$ such that any $v \in V$ can be joined to $w$ by a path in $U$. By composing, we have a path from $v$ to $x$ in $U$ and so, $v \in W$. Now take $w_{1}, w_{2} \in W$ and let $\gamma$ be a path in $U$ which joins the two. Then, for any $z \in \gamma$ we can join to $x$ in $U$ by using part of $\gamma$ and a path from $w_{1}$ to $x$ in $U$. Thus, $\gamma \subset W$ which completes the proof. 
We can also apply Proposition 2.1 to get a result for spheres in the case that the map has degree zero.

Corollary 2.4. Let $f: S^{k} \rightarrow S^{k}$ be a map of the $k$-sphere $S^{k}$ which is minimal and has distance from the constant map at the south pole less than $\pi / 2$. Then there is a Wecken homotopy which connects $f$ to the constant map $c(x)=s_{p}$.

Proof. Consider the restriction of the map $f$ to the closed southern hemisphere. Since the southern hemisphere is homeomorphic to the closed disk, we can apply Proposition 2.1. So it suffices to extend this homotopy to the northern hemisphere. Since the image of the northern part is contained inside of the southern hemisphere, we can extend so that the image lies inside the southern hemisphere. Hence, we have not created any new fixed points and the result follows.

We remark that there is a slightly different version of Corollary 2 which appears in [5, Section 3]. In that result, the maps $f_{1}$ and $f_{2}$ each has degree one. We assume that each of the two maps has distance less than $\pi$ to the identity. The proof given there is independent of Proposition 2.1. These two results give evidence that the space $C_{\min }\left(S^{k}, f\right)$ may be locally path connected.

\section{Connectedness properties for spheres}

In this section, we present similar results concerning path connectedness for the space $C_{\min }\left(S^{k}, f\right)$ where $S^{k}$ is the sphere of dimension $k$. The result given in Section 2 was only for maps of degree zero. Another special case occurs when the degree is $(-1)^{k+1}$, that is, when $f$ is fixed point free. Using the geometry of the sphere, it is a direct argument to verify both path connectedness and local path connectedness. For example, the unique geodesic path in $S^{k}$ joining $f(x)$ to $-x$ can be used to construct a Wecken homotopy between the fixed point free map $f$ and the antipodal map.

For the results in this section, there will be no dependence on the degree of the map, but our considerations will be restricted to dimensions 1, 3, and 7. Due to the existence of a multiplication structure, these problems can be addressed. We first prove the following theorem.

Theorem 3.1. If $k=1,3$ or 7 , then $C_{\min }\left(S^{k}, f\right)$ is path connected.

We remark here that the number of fixed points for minimal maps on spheres is given by the Nielsen number. In particular, when $k>1$ this is 1 if the map is not homotopic to the antipodal map and is 0 , otherwise. For $k=1$, this reduces to $|1-d|$ where $d$ is the degree of the map (see [8, Theorem 6.3]).

To prove this theorem, we first need a preliminary result given in Proposition 3.2. This was proved in [5, Propositon 1.4] in the case of the 2-sphere. That proof easily adapts to spheres of higher dimension. Recall that a root of a map $f: X \rightarrow Y$, where the space $Y$ has a base point $y_{0}$, is an element of $f^{-1}\left(y_{0}\right)$, that is, an element of the preimage of $y_{0}$ by $f$. 
Proposition 3.2. Let $f_{1}, f_{2}: S^{k} \rightarrow S^{k}, k \geq 2$, be two homotopic maps with $\operatorname{deg}\left(f_{1}\right)=\operatorname{deg}\left(f_{2}\right) \neq 0$ and suppose each map has exactly one root. Then, there is a homotopy connecting them, where the homotopy has one root at each stage. Moreover, if $p$ is the root for each $f_{i}$, then we can arrange that $p$ is the root for each level of the homotopy.

Proof. We give an outline of the proof from [5]. Using an isotopy of the identity, we first arrange that the maps have the same point as the root denoted by $p$. Since they have the same degree, we can make the two maps coincident in a small open neighborhood of $p$. On the complimentary $k$-ball, the two maps are homotopic as maps relative to the boundary with image in $S^{k}-p$. Any such homotopy gives the desired result.

Proof of Theorem 3.1. We first suppose that $k$ is either 3 or 7 . Let $g_{1}, g_{2}: S^{k} \rightarrow$ $S^{k}$ be two homotopic minimal maps. Consider the maps $f_{1}$ and $f_{2}$ defined by multiplication $f_{i}(x)=g_{i}(x)\left(x^{-1}\right)$. This multiplication is well defined and each element has a unique inverse [15, page 108].

If $g_{i}$ is not homotopic to the antipodal map, then each has exactly one fixed point. In this case, each $f_{i}$ has exactly one root at the identity of the multiplication. By Proposition 3.2, we have a homotopy $\left\{f_{t}\right\}, 1 \leq t \leq 2$, such that each $f_{t}$ has one root at 1 . It follows immediately that the map $g_{t}$ is well defined and that it has exactly one fixed point. In the case that each $g_{i}$ is fixed point free, we get that each $f_{i}\left(S^{k}\right)$ does not intersect the identity for the multiplication. Thus, the image lies in a ball and so we have a homotopy $f_{t}$ missing the identity.

Finally, in the case $k=1$, using $f_{i}$ just as above, we see that $\operatorname{deg}\left(f_{i}\right)=\operatorname{deg}\left(g_{i}\right)-$ 1. Thus, $f_{i}$ has exactly $\left|\operatorname{deg}\left(f_{i}\right)\right|$ roots at 1 . Apply Proposition 3.3 given below to obtain the desired homotopy.

Proposition 3.3. Let $f_{0}, f_{1}: S^{1} \rightarrow S^{1}$ be maps each of degree $d$ and each has exactly $|d|$ roots at 1 . Then, there is a homotopy between $f_{0}$ and $f_{1}$ such that each level map has exactly $|d|$ roots at 1 .

Proof. If $d=0$, this is straightforward, so we assume $d \neq 0$. We define the homotopy $f_{t}$ as follows. First on $S^{1} \times\{0,1\}$, we have the maps $f_{0}$ and $f_{1}$. In $S^{1} \times[0,1]$ choose a family of pairwise disjoint $\operatorname{arcs} \gamma_{1}, \ldots, \gamma_{|d|}$ such that for each $t \in[0,1]$, each of the arcs meets $S^{1} \times\{t\}$ in exactly one point; for $t=0$, the union is exactly $f_{0}^{-1}(1)$ and for $t=1$ it is $f_{1}^{-1}(1)$.

Consider a component $C$ of $\left(S^{1} \times[0,1]\right) \backslash\left(\cup \gamma_{i}\right)$. Let $C_{t}=C \cap\left(S^{1} \times\{t\}\right)$. For $i=0,1$, the endpoints of $C_{i}$ are mapped to 1 while the interior is sent to $S^{1}-1$. Now, each $f_{i}, i=0,1$ has the minimal number of roots in the given homotopy class (see [10, Example 3, page 127]). As a result, the two maps $f_{0}$ restricted to $C_{0}$ and $f_{1}$ restricted to $C_{1}$ are homotopic. Thus, we can extend to $C$ so that for each $t$, the endpoints of the $\operatorname{arc} C_{t}$ are mapped to 1 and the interior to $S^{1}-1$. Applied to each of the components, $C$ yields the desired homotopy.

Now consider local connectivity for spheres in these dimensions. 
Proposition 3.4. Let $k=1,3$, or 7 and let $f: S^{k} \rightarrow S^{k}$ be a map with exactly $N(f)$ fixed points. Given $\epsilon>0$, there exists $\delta>0$ such that for any map $g$ homotopic to $f$ with $N(f)$ fixed points, and such that the distance between $f$ and $g$ is less that $\delta$. There is a Wecken homotopy such that each level map is distance less than $\epsilon$ from $f$.

Proof. As in the proof of Theorem 3.1, we first consider the corresponding root problem. Its solution, then, translates via multiplication to the fixed point setting. For the case $k=3$ or 7 , we first observe that Proposition 3.2 admits a local version. Follow the proof of Proposition 2.1(B) to arrange that the maps have the same root, then extend in the complement using a small homotopy. The rest of the argument (following the proof of Theorem 3.1) is straightforward.

Now for the case of the 1-sphere and its local connectivity. As noted in the proof of Proposition 3.3, for a self-map of $S^{1}$ of degree $r$, the minimal number of roots is given by $|r|$.

Let $f, g: S^{1} \rightarrow S^{1}$ be two maps of degree $r$ each having $|r|$ roots at 1 and distance apart less than $\epsilon$. Let $N$ denote the open $\epsilon$-neighborhood of 1 and set $S=S^{1} \backslash N$. Throughout the argument, we will deform the map $g$ until the map $f$ is reached. At any time in the process, we let $\gamma_{x}$ denote the geodesic arc joining $f(x)$ and $g(x)$.

The first step is to deform $g$ along each $\gamma_{x}$ where $x \in f^{-1}(S)$ so that at the end, we have $g(x)=f(x)$ on $f^{-1}(S)$. Since each $\gamma_{x}$ has length less than $\epsilon$, this can be done by an $\epsilon$-homotopy. Also, this can be done without a change in the root set for $g$.

Now consider an interval $I$ in the compliment of $f^{-1}(S)$. Clearly, $f$ maps the endpoints of $I$ into the endpoints of $N$. Thus, $g$ does as well. With $\epsilon$ suitably small (say $\pi / 4$ ), it follows that $g$ maps $I$ into an interval containing $N$. In particular, if $I$ contains a root for $f$ (since $f$ is minimal, there is at most one root in $I$ ), then $g$ has a root in $I$. So, we have $|r|$ intervals each containing one root, each for $f$ and $g$.

First consider $I$ without any roots. Thus, both $f$ and $g$ map $I$ into one of the intervals in $N-1$, and so for each $x \in I, \gamma_{x}$ does not meet 1 . Deform $g$ along the geodesic to get $f(x)=g(x)$ on $I$.

Finally, for $I$ which has a root for each map deform $g$ by the following three steps:

(1) arrange that $g$ is one-to-one on $I$,

(2) by an isotopy of the interval $I$, arrange that $g$ has the same root point in $I$ as does $f$,

(3) for each $x \in I-f^{-1}(1), \gamma_{x}$ will miss 1 . Finish by deforming $g$ on each of these two intervals.

Since in each of (1), (2), and (3) the image of the deformation is always in $N$, we preserve the property that distance is less than $\epsilon$. Taking $\delta=\epsilon$, this completes the proof of the case $k=1$.

Corollary 3.5. The space $C_{\min }\left(S^{k}, f\right)$ is locally path connected for $k=1,3,7$. 


\section{Acknowledgments}

The second author would like to thank the Instituto de Matemática e Estatistica (IME) at Universidade de São Paulo for its hospitality during the preparation of this manuscript. This visit was supported by Fundaçao de Amparo à Pesquisa do Estado de Sāo Paulo (FAPESP).

\section{References}

[1] M. Bonino, L'espace des homéomorphismes de Brouwer est connexe par arcs [The space of Brouwer homeomorphisms is arcwise connected], C. R. Acad. Sci. Paris Sér. I Math. 321 (1995), no. 10, 1359-1361 (French).

[2] - A dynamical property for planar homeomorphisms and an application to the problem of canonical position around an isolated fixed point, Topology 40 (2001), no. $6,1241-1257$.

[3] D. Dimovski and R. Geoghegan, One-parameter fixed point theory, Forum Math. 2 (1990), no. 2, 125-154.

[4] R. Geoghegan and A. Nicas, Parametrized Lefschetz-Nielsen fixed point theory and Hochschild homology traces, Amer. J. Math. 116 (1994), no. 2, 397-446.

[5] D. L. Gonçalves and M. R. Kelly, Maps between surfaces and minimal coincidence sets for homotopies, Topology Appl. 116 (2001), no. 1, 91-102.

[6] Maps into the torus and minimal coincidence sets for homotopies, Fund. Math. 172 (2002), no. 2, 99-106.

[7] J. Jezierski, One codimensional Wecken type theorems, Forum Math. 5 (1993), no. 5, 421-439.

[8] B. J. Jiang, Lectures on Nielsen Fixed Point Theory, Contemporary Mathematics, vol. 14, American Mathematical Society, Rhode Island, 1983.

[9] M. R. Kelly, Some examples concerning homotopies of fixed point free maps, Topology Appl. 37 (1990), no. 3, 293-297.

[10] T.-H. Kiang, The Theory of Fixed Point Classes, Springer-Verlag, Berlin, 1989.

[11] F. Le Roux, Étude topologique de l'espace des homéomorphismes de Brouwer. I [Topological study of the space of Brouwer homeomorphisms. I], Topology 40 (2001), no. 5, 1051-1087 (French).

[12] __ Étude topologique de l'espace des homéomorphismes de Brouwer. II [Topological study of the space of Brouwer homeomorphisms. II], Topology 40 (2001), no. 5, 1089-1121 (French).

[13] C. R. F. Maunder, Algebraic Topology, Van Nostrand Reinhold, London, 1970.

[14] B. V. Schmitt, L'espace des homéomorphismes du plan qui admettent un seul point fixe d'indice donné est connexe par arcs, Topology 18 (1979), no. 3, 235-240 (French).

[15] N. Steenrod, The Topology of Fibre Bundles, Princeton Mathematical Series, vol. 14, Princeton University Press, New Jersey, 1951.

Daciberg L. Gonçalves: Departamento de Matemática, Instituto de Matemática e Estatistica, Universidade de São Paulo (IME-USP) Caixa Postal 66281, São Paulo, SP, Brazil

E-mail address: dlgoncal@ime.usp.br

Michael R. Kelly: Department of Mathematics and Computer Science, Loyola University, 6363 St. Charles Avenue, New Orleans, LA 70118, USA

E-mail address: kelly@loyno.edu 


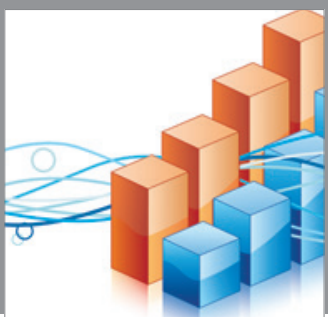

Advances in

Operations Research

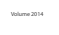

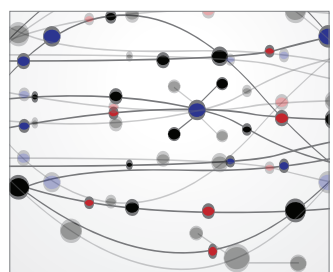

\section{The Scientific} World Journal
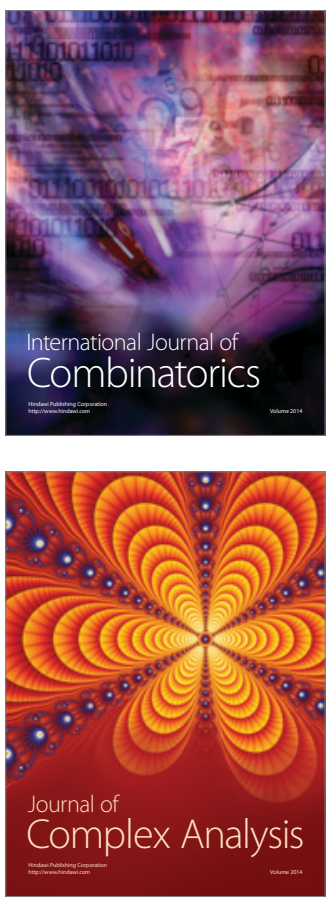

International Journal of

Mathematics and

Mathematical

Sciences
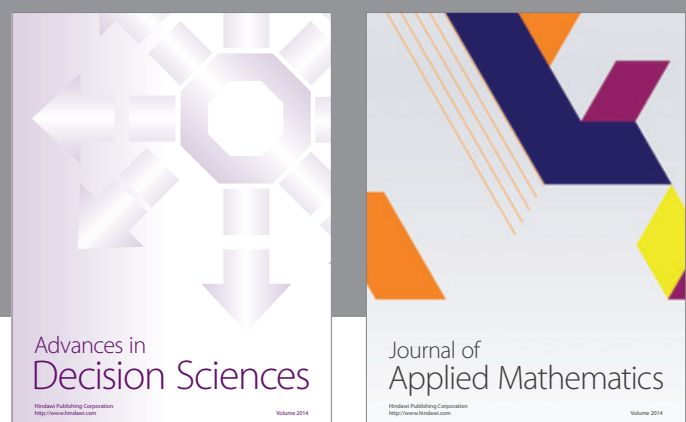

Journal of

Applied Mathematics
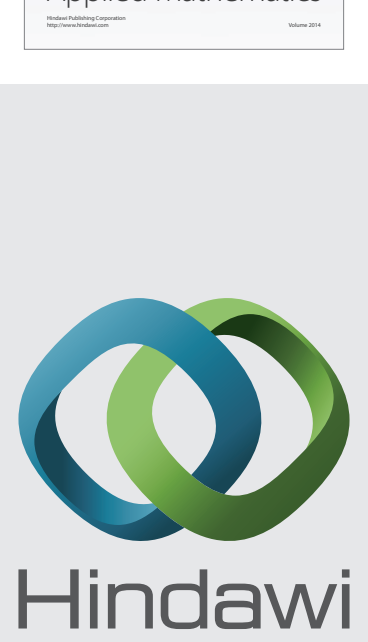

Submit your manuscripts at http://www.hindawi.com
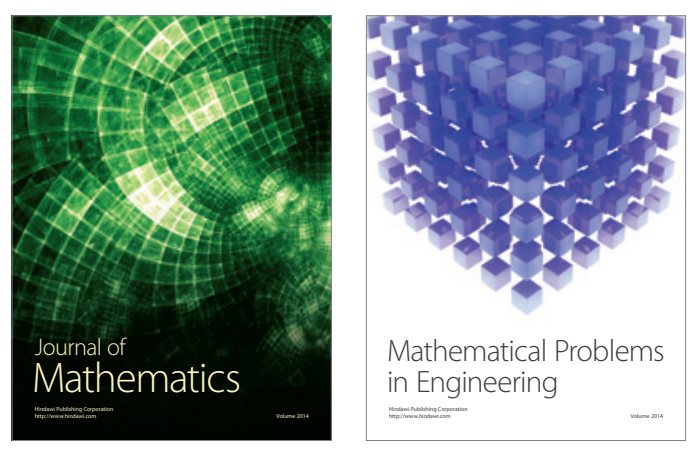

Mathematical Problems in Engineering
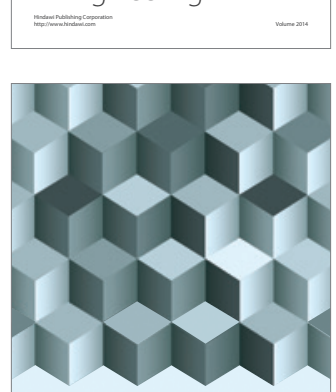

Journal of

Function Spaces
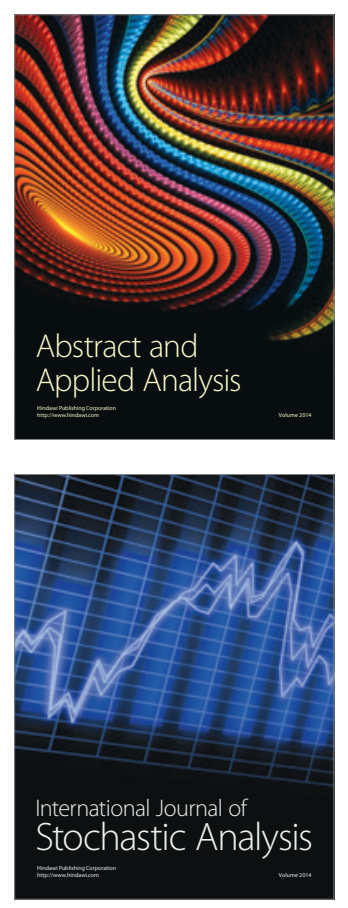

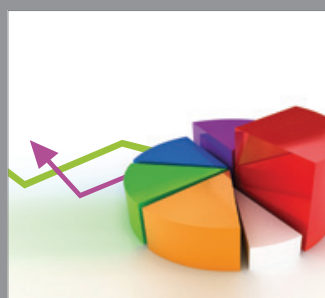

ournal of

Probability and Statistics

Promensencen
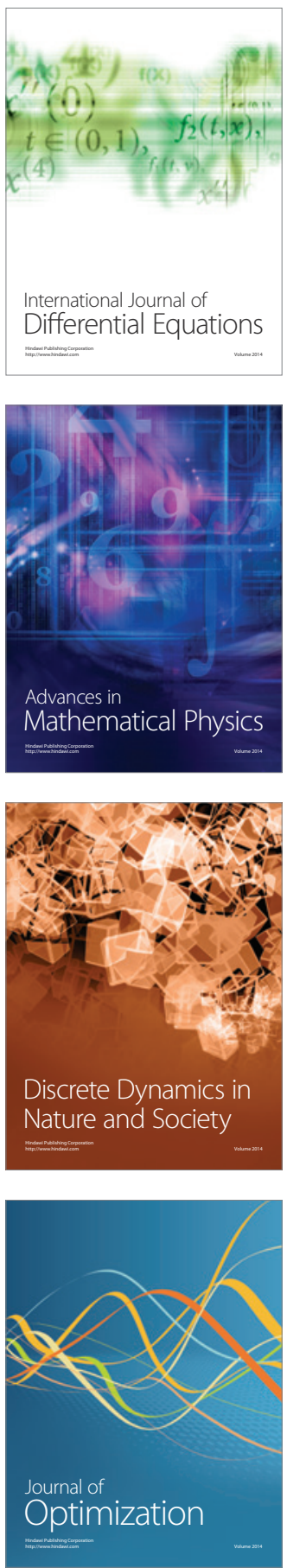